\title{
Open Ended Microwave Oven for Flip-Chip Assembly
}

\author{
K.I. Sinclair ${ }^{\sharp 1}$, A.J. Sangster ${ }^{\sharp 2}$, G. Goussetis ${ }^{\# 3}$, M.P.Y. Desmulliez ${ }^{\# 4}$, T. Tilford $^{* 1}$, A.K Parrott ${ }^{* 2}$ and C. Bailey ${ }^{* 3}$ \\ \# MicroSystems Engineering Centre (MISEC), Heriot Watt University, \\ Riccarton, Edinburgh, EH14 4AS, Scotland, UK \\ ${ }^{1} \mathrm{~K}$. Sinclair@hw.ac.uk \\ ${ }^{2}$ A.J.Sangster@hw.ac.uk \\ ${ }^{3}$ G. Goussetis@hw.ac.uk \\ "M.Desmulliez@hw.ac.uk \\ ' Centre for Numerical Modelling and Process Analysis, University of Greenwich, \\ Greenwich Campus, Old Royal Naval College, Park Row, London, SE10 9LS \\ ${ }^{1}$ t.tilford@gre.ac.uk
2a.k.parrot@gre.ac.uk
${ }^{3}$ c.bailey@gre.ac.uk
}

\begin{abstract}
A novel open-ended microwave oven in the form of a waveguide cavity partially filled with dielectric is proposed for the microwave curing of bumps, underfills and encapsulants during flip-chip assembly. By adjusting the dimensions and the dielectric permittivity, a well defined resonance can be confined in the dielectric part with non-radiating evanescent decaying fields in the remaining of the cavity. Curing occurs by virtue of the energy stored in localized evanescent field maxima. The dielectric to air interface enhances the longitudinal electric field and therefore the cavity is designed to operate at a TM mode. Careful selection of the resonance order can control the locations of the electric field maxima (hot-spots) allowing for spatially selective heating. The open end design offers enhanced flexibilities for the simultaneous curing and alignment of devices for fast flip-chip assembly, direct chip attach (DCA) or waferscale level packaging (WSLP). Low power tests using heat sensitive film demonstrate clearly that selective heating in multiple locations in the open end of the oven is achievable.
\end{abstract}

\section{INTRODUCTION}

Microwave power fundamentally accelerates the cure kinetics of polymer adhesives [1]. It provides a route to deposit heat energy exclusively into the polymer materials. Therefore microwaves can be used to minimise the temperature increase in the surrounding materials such as the substrate and die during the cure process. This is especially important for devices incorporating either low thermal budget materials or interfaces with large thermal coefficient mismatch. The concentration of heat into the polymer during the cure process promotes its adhesion properties, as the magnitude of residual stress between the polymer and materials, to which it is being bonded, will be low. For example, in flip-chip packages, delamination between encapsulant/underfill and the die is a major contributor to low reliability (hence lifetime) of such packages.

Current Variable Frequency Microwave (VFM) curing methods in electronic packaging bulk heat the entire package

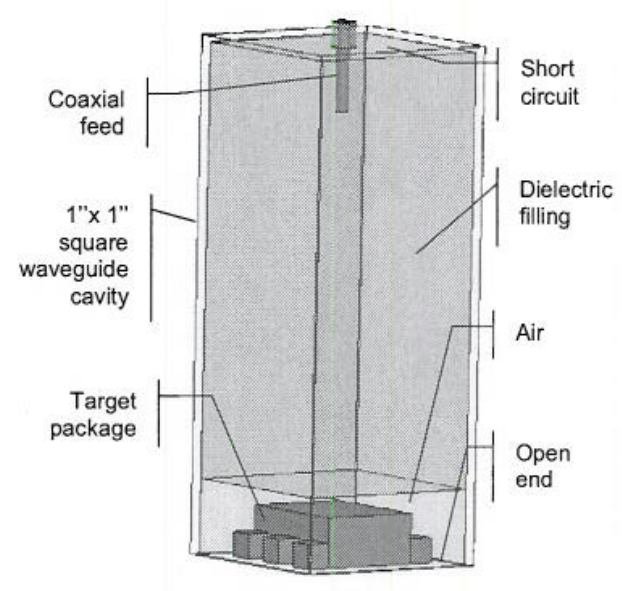

Fig. 1 Schematic layout of the open ended oven concept

or substrate [2]. Moreover, microwave heating cannot be performed today simultaneously with fine placement or alignment of the die or wafer onto the board. Instead significant delays are introduced in inserting and removing the device from the oven. This lack of capability reduces further the packaging throughput.

In order to address these limitations, this paper describes an open-ended oven which permits the package being cured to be accessed during the heating process. The simultaneous fine placement and curing of the device into a larger assembly creates productivity gains by combining assembly, placement and bonding into a single processing step. Moreover, by carefully selecting the transverse order of the resonance, predefined localised electric field maxima (hot-spots) can be produced. High order mode operation combined with suitable 
mode excitation schemes and frequency stepping offers the possibility of pre-selection of hot spots at the open end of the cavity to suit particular requirements of spatially controlled curing. The open-ended oven curing is achieved by exposing the target package to the non-radiating evanescent fields close to the 'open' exterior surface of a dielectric filled waveguide cavity resonator [3]. While it is well known that evanescent fields have been used to generate electromagnetic coupling in a variety of microwave devices [4], the use of such fields for microwave heating is novel to the best of the authors' knowledge.

This contribution demonstrates the principle of operation of the proposed microwave open oven. Full wave Finite Element Method (FEM) [5] is employed for the design and rigorous characterisation of a prototype. Measurements with a fabricated prototype oven operating at a high order mode in order to create multiple hot spots in the open end are also presented for validation.

\section{OPEN-ENDED MicROWAVE OVEN}

In electromagnetic terms, the microwave oven can be described as a terminated waveguide transmission line resonator. Because of radiation loss which normally occurs from the open end of a waveguide, empty metal waveguide cavity resonators are usually formed with short circuits at each end of the waveguide and thus the cavity takes the form of a closed box (if the waveguide has rectangular cross-section). However if the waveguide is dielectric filled, with a high permittivity, very low loss material, a resonant cavity is formed even if one end is 'open'. Radiation losses can be minimised if in the air region the resonant modes are evanescent. The resonant modes can then be approximated by trapped waves between the short circuit at one end and a quasi-open circuit formed by the dielectric-air interface at the other end.

At the open end of the cavity the transverse magnetic field components must equal zero (assuming a perfect open circuit) due to boundary conditions. In practice these fields are small but finite since a perfect open-circuit is not possible. If the open end represents a 'good' open circuit to the cavity fields the only significant fields to exist there are the transverse (to the guide axis) electric fields, which are close to their maximum values. Continuity of tangential electric fields at a dielectric interface requires that similar strength fields will exist at the external surface of the dielectric, essentially in the form of stored energy. A sample of lossy material, placed in these fields, will experience ohmic heating if sufficient power is supplied. The open-ended cavity becomes an open-oven. A schematic representation is shown in Fig. 1.

The quality of the 'open-circuit' is dependent of the magnitude of the relative permittivity of the dielectric. If it is to be viable, the open-ended oven requires the permittivity of the filling material to be high enough to encourage strong modal resonances by inhibiting radiation from the open-end. Moreover, the boundary conditions in the dielectric-air interface,

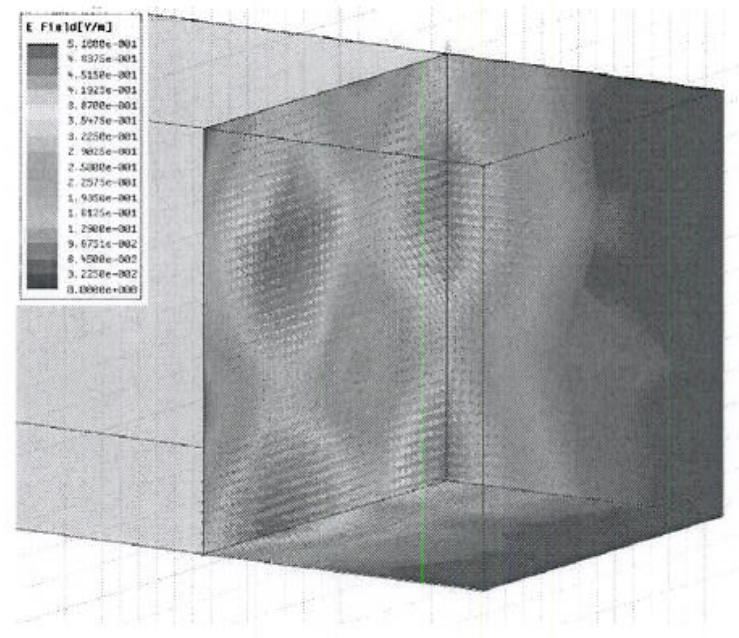

Fig. 2 Evanescent Fringing Field within cut-off region

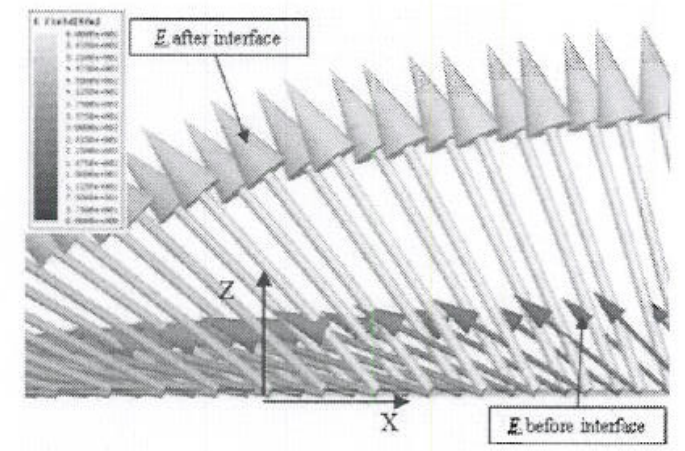

Fig. 3 FEM model of the total electric field distribution at the dielectric interface (dark arrows = just inside interface; light arrows $=$ just on the air side of the interface)

$$
\begin{aligned}
& E_{n 2}=\varepsilon_{r} E_{n 1} \\
& E_{t 2}=E_{t 1}
\end{aligned},
$$

where $E_{n l}$ and $E_{t l}$ are the normal and tangential electric fields at the interface in the dielectric region and $E_{n 2}$ and $E_{12}$ are the corresponding quantities in the air region, suggest that the longitudinal $E$-field component in the air side of the discontinuity is multiplied by a factor equal to the permittivity of the cavity filling. To exploit this field enhancement in the air region, it is here proposed that the oven operates on a TM resonant mode. The order of the mode is chosen to selectively concentrate the hot-spots in the localities of the curing polymers.

\section{NUMERICAL RESULTS}

A design and performance assessment of the open-oven concept was carried out by modelling a prototype design on a full wave electromagnetic simulation package [5]. The dimensions of the cavity are $24 \mathrm{~mm} \times 24 \mathrm{~mm} \times 110 \mathrm{~mm}$. The 


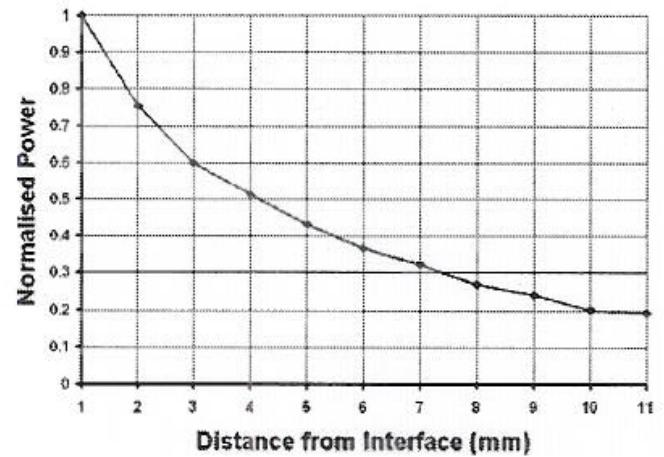

Fig 4 Variation in absorbed power within the pellet with distance from interface

ceramic oven filling material was modelled with a relative permittivity of 6 and a $\tan \delta$ of 0.006 . Also the filling was made $5 \mathrm{~mm}$ shorter than the length of the waveguide to procure a short section of cut-off waveguide at the open-end. In the 'driven mode' of operation the model was excited at the short circuit end with a $z$-directed probe to ensure that only TM modes were set up.

Fig. 2 displays $|E|$ within the cut-off section (air) of waveguide. One quarter section has been simulated incorporating two perfect magnetic wall symmetry planes in order to reduce the computational effort. The $\mathrm{TM}_{33,5 / 2}$ mode exhibits a strongly selective evanescent field within the cut-off section. The field tends to $0 \mathrm{~V} / \mathrm{m}$ after approximately $3 \mathrm{~mm}$.

Fig. 3 plots a detail of the electric fields in the planes immediately before and after the dielectric-air interface. It is clear from Fig. 3 that the FEM model predicts an electric field at the air/dielectric interface which is considerably stronger on the air side of the interface than at the interface inside the dielectric. The levels are in good agreement with the predictions made from the electric flux density continuity relationship (1).

\section{POWER DISSIPATION}

The heating effect of the evanescent fields on the air side of the dielectric interface at the open end of the oven has also been modelled by inserting a lossy pellet $(1.0 \mathrm{~mm}$ radius, $0.2 \mathrm{~mm}$ thickness, $\varepsilon_{r}=3.96, \rho=75.76 \mathrm{ohms}-\mathrm{m}$ ) in a region of high field. This pellet is considered to be typical of a volume of paste requiring curing within an integrated circuit package. The power absorbed by the pellet $\left(P_{a}\right)$, since it is small relative to the wavelength, is calculated using the approximate relation,

$$
P_{n}=\frac{|E|^{2}}{2 \rho} \times \text { volume Watts }
$$

$P_{a}$, normalised to the maximum value when the pellet is adjacent to the dielectric interface, is plotted as a function of axial distance $(z)$ from the interface in Fig. 4. As anticipated the power absorbed decays exponentially with distance due to the evanescent nature of the external field. However the rate of decay, in this case for a mode operated not too far from cutoff, is conducive to manageable open-end heating. At

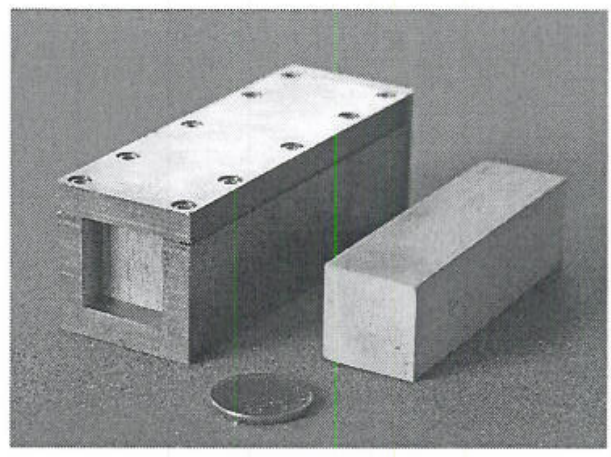

Fig 5 Open cavity oven. The dielectric material filling most of the cavity is presented on the right.

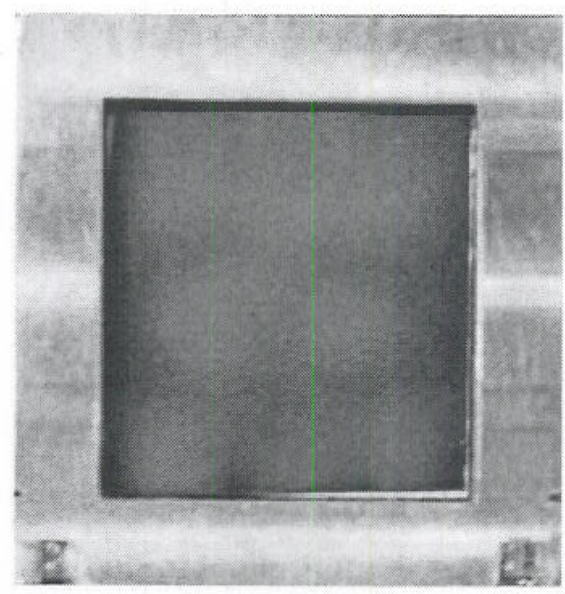

Fig 6 Change in heat distribution on heat sensitive film placed at dielectric interface

$z=0.1 \mathrm{~mm}$ (pellet in contact with the interface) the power absorbed by the pellet is $16 \mu \mathrm{W}$ for an oven input power of $1 \mathrm{~W}$. For this prototype most of the supplied power is dissipated in the ceramic filling, which has a $\tan \delta=0.006$. Considerably more power could, of course, be extracted from the oven if the pellets occupied a much larger volume of the evanescent fields. In the next generation prototype ceramic filling materials exhibiting much lower $\tan \delta$ values will be used. Nevertheless, the pellet power absorption of $16 \mu \mathrm{W}$ for $1 \mathrm{~W}$ of input power suggests that in the current relatively inefficient prototype, an oven power of $300 \mathrm{~W}$, for about 1 minute, would be sufficient for curing a typical IC package in the open-ended microwave oven.

\section{MeAsured Results}

A proof-of-concept prototype (Fig. 5) of the 'open-ended' oven has been fabricated and experimentally tested. It comprises a dielectric filled rectangular waveguide having a short circuit at the excitation end of the waveguide and an open circuit at the opposite end, such that the target package to which heat is to be applied, can be placed easily within the 
TABLE I

MEASURED AND SIMULATED RESULTS

\begin{tabular}{|c|c|c|c|}
\hline $\mathbf{k}$ & $\begin{array}{c}\mathbf{f}_{\text {res }} \text { (GHz) } \\
\text { (Eigenmode) }\end{array}$ & $\begin{array}{c}\mathbf{f}_{\text {res }} \text { (GHz) } \\
\text { (HFSS) }\end{array}$ & $\begin{array}{c}\mathbf{f}_{\text {res }} \text { (GHz) } \\
\text { (Measured) }\end{array}$ \\
\hline 0 & 10.036 & - & - \\
\hline $1 / 2$ & 10.042 & 10.01 & 9.905 \\
\hline $3 / 2$ & 10.079 & 10.045 & 10.00 \\
\hline $5 / 2$ & 10.153 & 10.125 & 10.12 \\
\hline
\end{tabular}

evanescent fields adjacent to the dielectric interface. The dimensions of the prototype are the same as those employed in the FEM model. The prototype described in this paper was specifically designed to provide a demonstration of the formation of multiple 'hotspots' in the open-end. A photograph of the fabricated prototype is shown in Fig. 5. Measurements with heat sensitive film inserted in the recess and on the surface of the dielectric are shown in Fig. 6. The temperature sensitive film is designed to display black at $24^{\circ} \mathrm{C}$, red at $26^{\circ} \mathrm{C}$, green at $28^{\circ} \mathrm{C}$ and blue at $30^{\circ} \mathrm{C}$. These measurements indicate that the $\mathrm{TM}_{3,3,5 / 2}$ mode is present at $10.12 \mathrm{GHz}$. Measured and predicted resonant frequencies are shown in table 1 . The system was powered from a travellingwave tube capable delivering approximately 15 Watts to the cavity to obtain this result.

It is clear that the measured mode shown in Fig. 5 is not a 'pure' $\mathrm{TM}_{3,3,3 / 2}$ mode as anticipated. This was true of other measurements on other modes. Only 8 rather than 9 pattern peaks can be discerned for the $\mathrm{TM}_{3,3,3 / 2}$ mode. Further testing has shown that in the practical cavity the $\mathrm{TM}_{3,3,5 / 2}$ mode is quasi-degenerate with the $\mathrm{TM}_{4,1,7 / 2}$ mode. The quasidegeneracy is attributable to the fact that the available Qlevels were insufficiently high, causing the modal resonances of neighbouring modes to overlap. The measured $\mathrm{Q}$ levels were in the range 200-300 for the $\mathrm{TM}_{3,3,5 / 2}$ mode.

This could be improved by employing a lower loss dielectric insert and higher conductivity metal cavity walls. Quasi-degeneracy can also be suppressed by employing mode selective coupling involving the use of more than one excitation probe. The $\mathrm{TM}_{3,3, \mathrm{k}}$ mode oven with an 'open circuit' at the open end does not take full advantage of the strong axial electric field formed by the mode when operated not too far from cut-off. If the 'open circuit' were 'perfect', no normal component of $E$-field would exist at the interface. The normal $E$-field within a waveguide cavity maximises in theory on a perfectly conducting wall. Modelling of the cavity with a thin metal layer (thickness of $1 / 20$ skin depth) deposited on the open interface shows that a strong normal field does penetrate through the metal layer. This has been confirmed experimentally by inserting thin metallised glass slides in the open end of the cavity. Unfortunately the metal layer carries high currents at the power levels required for heating and could easily be burnt off. A more sophisticated impedance wall which behaves like a quasi-short-circuit to the transverse fields at the open end of the oven while permitting the normal electric field component at its surface to pass through with minimal attenuation is required to make it possible to demonstrate more efficient heating at high power levels.

\section{CONCLUSION}

A novel microwave oven for the fixed frequency curing of Isotropically Conductive Adhesives (ICA) bumps at high alignment accuracies has been presented. The intent is to insert the proposed oven into a flip-chip bonding machine such that high alignment accuracy can be achieved and the heating of ICA bumps done in situ and simultaneously. This would lead to an increased throughput in packaging. The findings of the simulations have been demonstrated through the manufacturing of a proof-of-principle test vehicle. Preliminary experiments demonstrate that effective heating takes place in accordance with the simulated results. Further work is ongoing to demonstrate the adhesion strength of the bonded devices.

\section{ACKNOWLEDGEMENTS}

The authors would like to acknowledge the financial support of the EEC-funded Network of Excellence Patent-DfMM (Design for Manufacture of Microsystems), the EPSRCfunded Innovative electronic Manufacturing Research Centre through the grant number FE/05/01/07 and the EPSRC-funded Scottish Manufacturing Institute (SMI).

\section{REFERENCES}

[1] T. Wang, Y. Fu, M. Becker, M. Zhou, and J. Liu, "Microwave Heating of Metal-Filled Electrically Conductive Adhesive Curing," IEEE Electronic Components and Technology Conference 2001, pp.593-597, Jun. 2001 .

[2] Lauf, R .J., Bible, D. W., Hohnson, A. C. and Everleigh C. A., "2 to 18 Broadband microwave Heating Systems," Microwave Journal, Nov. 1993, pp.24-34.

[3] K. I. Sinclair, M.P.Y. Desmulliez, A.J. Sangster, "A novel RF-curing technology for microelectronics and optoelectronics packaging", I" Electronics Systemintegration Technology Conf., Dresden, Germany, 5-7 Sept., 2006.

[4] J.K. Plourde, C-L. Ren, C-L., "Application of dielectric resonators in microwave components", IEEE Trans. Microw. Theory and Tech., Vol. MTT-29, No. 8, pp754-770, 1981

[5] [2007] Ansoft Corporation, High Frequency Structure Simulator [Online]. Available: http://www.ansoft.com 